six candidate genes by real-time polymerase chain reaction quantitation analysis of four GBM samples. Investigation of these genes should help provide important insights into the biological mechanisms at work, facilitate identification of tumors that are susceptible or resistant to radiation therapy and aid in the design of approaches to enhance specifically the radiosensitivity of these deadly neoplasms.

Grandori, Carla

\section{Gene expression profiles at various stages of lymphomagenesis in Em-myc transgenic mice}

Carla Grandori, Brian Iritani \& Robert Eisenman

Fred Hutchinson Cancer Research Center, Seattle, Washington, USA

To define molecular signatures of c-myc-induced B-cell neoplasias we have explored, using DNA microarrays, gene expression profiles at various stages of tumorigenesis utilizing the transgenic Em-myc model ${ }^{1}$. In this system c-myc is expressed, under the control of the immunoglobulin heavy-chain enhancer, selectively in B-cell precursors, and it causes the rapid appearance of B-cell lymphomas. Overt tumors are preceded by a marked polyclonal expansion of pre-B cells both in the bone marrow and in the spleen. However, these cells are not yet tumorigenic. Within a few months the Em-myc mice develop a monoclonal or oligoclonal lymphoma. We monitored gene expression changes using an 11,000-gene chip (Affymetrix) of pre-B cells derived from the bone marrow of Em-myc mice before tumor development and cells from lymphomas, consisting primarily of pre-B cells. Both samples were then compared with normal pre-B lymphocytes obtained from bone marrow of wild-type mice. The data analysis allowed a display of gene expression changes that accompany these various stages of tumor development. Preliminary results indicate that specific signaling pathways are selectively altered in the tumor samples or in the hyperplastic pre$\mathrm{B}$ cells. In addition, comparison of the pre-B cells expressing Em-myc with wild-type pre- $\mathrm{B}$ cells highlighted gene expression changes that might be direct consequences of deregulated c-myc expression and therefore provided new candidate $M y c$ target genes. We will present a discussion of the relevance of our study for the understanding of $\mathrm{c}-m y c$-induced tumorigenesis.

1. Adams, J.M. et al. Nature 318, 533-538 (1985).

Graveel, Carrie

\section{Analysis of gene expression alterations in mouse and human hepatocellular carcinomas}

\author{
Carrie Graveel ${ }^{1}$, Tim Jatkoe ${ }^{2}$, Steve Madore ${ }^{2}$, Alison Holt ${ }^{1}$ \\ $\&$ Peggy Farnham ${ }^{1}$ \\ ${ }^{1}$ McArdle Laboratory for Cancer Research, University of Wisconsin-Madison, \\ Madison, Wisconsin, USA \\ ${ }^{2}$ Pfizer Global Research and Development, Ann Arbor, Michigan, USA
}

Liver cancer is the fifth most common cancer worldwide, with 437,000 cases reported in 1990. Yet a mutational model has not yet been developed for liver cancer, as it has for certain other cancers, such as colon cancer. A thorough understanding of the molecular events leading to neoplastic transformation of the liver requires a detailed comparison of the gene expression pattern in normal liver cells with that in cancer cells. We have performed gene expression profiling of normal and neoplastic livers. Using oligonucleotide microarrays, we compared liver tumors (from diethylnitrosamine-treated $\mathrm{C} 3 \mathrm{H} / \mathrm{HeJ}$ mice) with three different states of the normal liver: quiescent adult, regenerating adult and newborn.
Although each comparison revealed hundreds of differentially expressed genes, only 22 genes were found to be deregulated in the tumors in all three comparisons. We also employed representational difference analysis to clone fragments of messenger RNAs differentially expressed in liver tumors versus regenerating livers. Although many of the same mRNAs were identified as in the oligonucleotide microarray experiments, we also cloned several new mRNAs that are differentially regulated in liver tumors. We have cloned the mouse complementary DNA of novel 4 and are currently isolating the human homologue of this unknown gene. We are using representational difference analysis and oligonucleotide microarrays to identify genes whose expression is deregulated in the development of human hepatocellular carcinomas. Using these models and techniques, we hope to identify common genetic alterations in the progression of liver cancer in both humans and mice.

Gregg, Jeff

$[3]$

\section{Molecular profiling of a mouse model for metastatic breast carcinoma}

\author{
Katti Jessen, Stephenie Liu, Larry Yong, Robert Cardiff \& \\ Jeffrey Gregg
}

Department of Pathology, University of California Davis School of Medicine, Sacramento, California, USA

Breast cancer is among the most common human cancers. One of the significant predictors of prognosis is distant metastasis. As cancer is the consequence of a broad dysregulation of cell signaling pathways, the ability of cells to metastasize may be due to changes in a limited number of pathways related to invasiveness and metastasis. Our study focuses on gaining a better understanding of these pathways using a mouse model for mammary tumor metastasis. We are working with two transplantable mouse mammary tumor lines with significant differences in metastatic potential. Met-1 tumors develop $100 \%$ pulmonary metastases while Db-7 tumors exhibit significantly fewer pulmonary metastases (9\%). In order to extract genes differentially expressed in both Met- 1 and Db-7, we performed two suppression subtractive hybridization (SSH) experiments. From each of the subtracted libraries, 2600 clones were PCR-amplified and arrayed. By fluorescently labeling the unsubtracted Met- 1 and Db-7 libraries and hybridizing them onto the microarray, we were able to demarcate the truly differentially expressed genes. Clones found to be greater than two-fold differentially expressed were then sequenced for identification. Differential expression of each gene was verified by RT-PCR, Northern blot, and Western blot. In order to determine the function of a subset of these genes, anti-sense and sense constructs were introduced into cell lines derived from these tumors. Migration assays were performed and the metastatic potential of each gene was assessed. We have identified several interesting genes that are both differentially and functionally important for the metastatic phenotype in this model.

Hager, Jeff

\section{Genomics of islet cell carcinogenesis}

Jeff Hager ${ }^{1}$, Graeme Hodgson ${ }^{1}$, Colin Collins ${ }^{1}$, Fong-Ying Tsai $^{2}$, Dennis Huszar ${ }^{2}$, Joe Gray ${ }^{1} \&$ Douglas Hanahan ${ }^{1}$

${ }^{1}$ University of California San Francisco, San Francisco, California, USA ${ }^{2}$ Millennium Pharmaceuticals, Cambridge, Massachusetts, USA

All cancers are thought to develop through a series of distinct stages, which are the result of an accumulation of both genetic and epigenetic changes. This is evident in a mouse model of pancreatic islet cell tumorigenesis, RIPTag, in which the focal nature of the endocrine pancreas has allowed accurate identification, quantifica- 


\section{Abstracts: Session II}

tion and physical isolation of such stages. All islets express the oncoprotein $\mathrm{T}$ antigen, but with decreasing frequency in hyperplastic islets (50-70\%), angiogenic islets $(10 \%)$ and end-stage tumors (2-4\%). A long-term goal of our studies is to identify the genetic and epigenetic alterations that are required at each of these steps en route to tumor formation. Two genetic alterations have been identified in this model in a genome-wide screen for regions of loss of heterozygosity (LOH). One resides on chromosome 16 ( $\mathrm{LOH16}$ ) and is lost in the transition between hyperplastic islet and angiogenic islet. The other is on chromosome 9 ( $\mathrm{LOH} 9)$ and is lost as angiogenic islets develop into tumors. To identify the tumor suppressor genes contained within $\mathrm{LOH} 9$ and $\mathrm{LOH} 16$, we are mapping the fine structure of the two regions by analysis of simple sequence length polymorphisms and comparative genomic hybridization with BAC-based arrays. We have developed and validated the use of murine BACs spotted on glass slides as a sensitive platform for comparative genome hybridization, with which we can readily detect loss of $\mathrm{LOH} 9$ and $\mathrm{LOH}_{16}$ in islet tumors. To identify genes whose altered expression may be critical to tumor development we have also profiled expression of approximately 22,000 genes and expressed sequence tags on microarrays using messenger RNAs derived from distinct RIPTag tumor stages. We will present patterns of gene expression and potential candidates for functional studies.

He, Huiling

\section{Gene expression analysis of glucocorticoid- induced apoptosis by a gene microarray technique}

Huiling He, Michael Malone, Steven Ostrowski \& Clark Distelhorst

Case Western Reserve University, Cleveland, Ohio, USA

Glucocorticosteroids induce apoptosis in immature lymphocytes and are therefore commonly used to treat patients who have lymphoid leukemia or lymphoma. To understand the genetic pathways and molecular mechanisms of glucocorticoid-induced apoptosis, we used the gene microarray technique to analyze gene expression in a well-established mouse lymphoma cell line, S49.1. In initial experiments S49.1 cells were treated with the glucocorticoid hormone, dexamethasone or vehicle for $12 \mathrm{~h}$, and total RNA was extracted. Biotinylated antisense RNAs were hybridized to Affymetrix mouse U74 sub A chips, which contain about 12,000 known genes and expressed sequence tags. Following dexamethasone treatment, 69 genes had variances greater than 2 -fold, and 40 genes had variances greater than 2.5 -fold. Among these 40 genes, 30 were induced and 10 were repressed in the dexamethasone-treated cells. Included among the known genes with altered expression were members of the immunoglobulin superfamily, transcription factors and receptors coupled to $G$ protein. Among the expressed sequence tags with altered expression were tags with a high degree of homology to potassium channel proteins and $\beta$-catenins or other new genes. Earlier time points of the gene expression patterns will be analyzed by the same approach in anticipation of finding other genes with potential involvement in the apoptosis pathway induced by glucocorticoids.
Hedenfalk, Ingrid

[6]

\section{Classification of hereditary breast cancers based on gene expression}

Ingrid Hedenfalk ${ }^{1,2}$, Yidong Chen ${ }^{1}$, David Duggan ${ }^{1}$, Åke Borg ${ }^{2} \&$ J effrey Trent ${ }^{1}$

${ }^{1}$ Cancer Genetics Branch, National Human Genome Research Institute, National Institutes of Health, Bethesda, Maryland, USA

${ }^{2}$ Department of Oncology, Lund University Hospital, Lund, Sweden

A large number of hereditary breast cancers are due to mutations in the two breast cancer susceptibility genes, $B R C A 1$ and $B R C A 2$. However, many hereditary breast cancer cases cannot be accounted for by mutations in these genes, and these are believed to be due to as yet unidentified breast cancer predisposition genes $(B R C A x)$. Using complementary DNA microarrays to classify hereditary breast cancers, we show that $B R C A 1$ and $B R C A 2$ tumors display distinct gene expression profiles and can be classified on the basis of gene expression patterns. We are analyzing several hereditary breast cancers that have tested negative for mutations in BRCA1 and BRCA2. We tested primary tumors against a microarray of 6,512 genes and employed mathematical analyses to determine a set of genes capable of discriminating mutation-positive breast cancers. Using permutation analysis of multivariate classification functions, we established that the expression profiles of $B R C A 1$ and $B R C A 2$ tumors were significantly different statistically. With analysis of variance between gene expression levels and genotype we ascertained those genes capable of separating BRCA1 from BRCA2 breast tumors. Examination of this gene list suggested functional differences between $B R C A 1$ and $B R C A 2$ tumors, including differences in $\mathrm{p} 53$ regulation and homeostasis, and (for BRCA1) the possible establishment of a constitutive stress-type state. We extended our gene expression results to encompass pathological findings by generating a tissue microarray of approximately 200 breast tumor specimens. We are currently determining if $B R C A x$ breast tumors can be classified and subjected to linkage analysis to recognize new breast cancer predisposition loci. Gene expression analysis has for the first time revealed a consistent and significant gene expression difference between $B R C A 1$ and $B R C A 2$ cancers, a finding with therapeutic implications.

Hegi, Monika

[7]

\section{Molecular pathways in human glioblastoma assessed by gene expression profiling}

Monika Hegi ${ }^{1}$, Michimasa Nozaki ${ }^{1}$, Martin Labuhn², Roger Stupp ${ }^{1}$, Annie-Claire Diserens ${ }^{1}$, Marie-France Hamou ${ }^{1}$, Adrian Merlo ${ }^{2}$, Nicolas de Tribolet $^{1} \&$ Sophie Godart ${ }^{1}$

${ }^{1}$ University Hospital, Lausanne, Switzerland

${ }^{2}$ University Hospital, Basel, Switzerland

Glioblastoma is the most malignant form of astrocytic brain tumor; patients have a mean survival time of one year after diagnosis. Extensive genetic analyses have recognized few genetic alterations and have not yielded any predictive factors. A more comprehensive approach includes analysis of differential gene expression, reflecting regulatory differences. We seek to identify differentially expressed genes relevant for tumor development or possessing predictive value, in order to gain insights into the molecular mechanisms of tumor progression and uncover new therapeutic targets. Using complementary DNA macroarrays (Clontech) we have constructed gene expression profiles in glioblastoma biopsies from a homogenous group of patients enrolled in a pilot clinical trial (concomitant and adjuvant temozolomide and irradiation for newly diagnosed glioblastoma). We subjected the expression profiles to cluster analysis to shed light on the mechanistic aspects of 